\title{
Nebular emission lines in IRAS 17347-3139
}

\author{
F. M. Jiménez-Esteban ${ }^{1}$, J. V. Perea-Calderón ${ }^{2}$, \\ O. Suárez ${ }^{3}$, M. Bobrowsky ${ }^{4}$, and P. García-Lario ${ }^{5}$ \\ ${ }^{1}$ Hamburger Sternwarte, Hamburg, Germany \\ email: francisco.jimenez-esteban@hs.uni-hamburg.de \\ ${ }^{2}$ European Space Astronomy Centre (ESAC), Madrid, Spain \\ ${ }^{3}$ Laboratorio de Astrofísica Espacial y Física Fundamental-INTA, Madrid, Spain \\ ${ }^{4}$ Computer Science Corporation/Space Telescope Science Institute, Baltimore MD, USA \\ ${ }^{5}$ ESAC, Research and Scientific Support Deparment of ESA, Madrid, Spain
}

\begin{abstract}
We report the detection of nebular emission lines in the optical and mid-infrared spectra of IRAS 17347-3139, a heavily obscured OH/IR star which may be rapidly evolving from the AGB to the PN stage. The presence of emission lines is interpreted as a clear indication that the ionization of its circumstellar envelope has already started. This source belongs to the rare class of objects known as 'OHPNe' displaying both $\mathrm{OH}$ maser and radio continuum emission. However, unlike the rest of stars in this class, prominent C-rich dust features are detected in its mid-infrared spectrum, which makes the analysis of this star particularly interesting.
\end{abstract}

Keywords. stars: AGB and post-AGB.

\section{Introduction}

As low- and intermediate-mass $\left(0.8-8 \mathrm{M}_{\odot}\right)$ stars evolve along the Asymptotic Giant Branch (AGB) in their process to become Planetary Nebulae (PNe) they experience strong mass loss (up to $10^{-4} \mathrm{M}_{\odot} \mathrm{yr}^{-1}$ ) which results in the formation of gas and dust circumstellar envelopes (CSEs) which sometimes may become thick enough to completely obscure the light coming from the central star in the optical at the end of the AGB phase, when the mass loss rate is maximum. In particular, O-rich stars evolving from the AGB to the PN stage develop a strong and characteristic double-peaked $\mathrm{OH}$ maser emission at $1612 \mathrm{MHz}$ at the end of their AGB lifetime which later disappears when the mass loss drops below a certain level as the star enters the post-AGB stage. An interesting and peculiar class of sources recently discovered is the one formed by the so-called 'OHPNe' (Zijlstra et al. 1989). OHPNe are heavily obscured O-rich transition sources which show both this $\mathrm{OH}$ maser emission as well as radio continuum emission, indicative of ongoing ionization in their circumstellar envelope. They have been proposed to be the "missing link' between high-mass O-rich AGB stars experiencing hot bottom burning and Type I PNe. These massive objects may evolve so fast that when the central star reaches the necessary temperature to ionize the envelope, this is still thick enough to prevent the detection of the PN in the optical. These massive AGB stars may never be detectable in the optical as classical PNe. Rather, they would evolve as 'infrared PNe'. Unfortunately, the number of OHPNe known is very small (a dozen sources) to derive firm conclusions on their nature and evolutionary stage. Here we report on the identification of nebular emission lines in IRAS 17347-3139 (= GLMP 591), another member of this class showing some peculiar observational properties.

\section{Observations}

The optical observations here presented were carried out in June 2003 using EFOSC2 while the mid-infrared observations were carried out in July 2003 with TIMMI2, both in- 

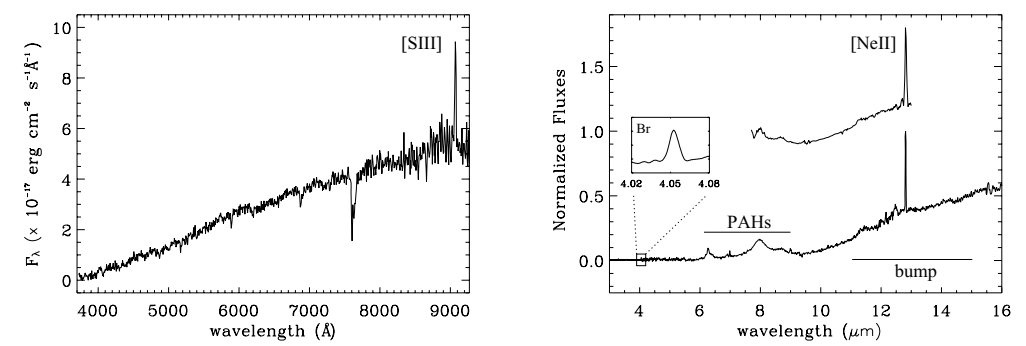

Figure 1. Left: The optical spectrum obtained with EFOSC2 at the ESO $3.6 \mathrm{~m}$ telescope, where the prominent [S III] 9068.6 $\AA$ emission is indicated. Right: ISO-SWS spectrum (bottom) showing $\mathrm{Br}_{\alpha}(4.05 \mu \mathrm{m})$ and [Ne II] $12.81 \mu \mathrm{m}$ emission lines. PAH emission features at $6.2,7.7$ and $8.6 \mu \mathrm{m}$ are also detected together with a broad plateau of hydrogenated PAHs extending from 11 to $15 \mu \mathrm{m}$. The detection of these features was confirmed with TIMMI2 (top)

struments installed at the ESO $3.6 \mathrm{~m}$ telescope in La Silla (Chile). The infrared spectrum covering the spectral range from 2 to $45 \mu \mathrm{m}$ was retrieved from the ISO Data Archive, and it was taken with the Short Wavelength Spectrometer in the SWS01 observing mode.

\section{Results}

The optical spectrum of IRAS 17347-3139 is shown in Figure 1 (left panel), where we can only see a very red continuum together with a strong [S III] $9069 \AA$ line in emission. In addition, $\mathrm{Br}_{\alpha}$ emission at $4.05 \mu \mathrm{m}$ and a prominent [Ne II] emission line at $12.81 \mu \mathrm{m}$ are clearly identified in the ISO SWS01 spectrum. The detection of [Ne II] was later confirmed in our mid-infrared observations with TIMMI2 (see Figure 1 right panel). This suggests that the ionization of the circumstellar shell has already started.

The detection of $\mathrm{OH}$ (Zijlstra et al. 1989) and $\mathrm{H}_{2} \mathrm{O}$ maser emission (Gregorio-Monsalvo et al. 2004) is a clear signature of the O-rich nature of the CSE surrounding this source. Unexpectedly, however, the mid-infrared spectrum shows the presence of broad and prominent solid state emission features at $6.2,7,7,8.6$ and $11.3 \mu \mathrm{m}$ usually attributed to Policyclic Aromatic Hydrocarbons (PAHs) in addition to a broad plateau of emission from 11 to $15 \mu \mathrm{m}$, characteristic also of hydrogenated PAHs (see Figure 1; right panel), which suggest that there is also a substantial amount of C-rich dust in the shell. This dual-dust chemistry has only been observed before in a few other transition sources and several hypothesis have been proposed to explain the phenomenon. Among them: a) a very recent thermal pulse which may have efficiently dredged up C-rich processed material to the stellar surface (Waters et al. 1998); b) a long-lived circumbinary disk which has preserved the O-rich material from destruction (Molster et al. 1999); and c) a result of the deactivation of hot bottom burning at the end of the AGB as a consequence of the strong mass loss (Frost et al. 1998). In order to determine which of the above hypothesis is correct additional observations are needed which should be able to reveal the relative distribution of O-rich and C-rich material within the circumstellar envelope.

\section{References}

Frost, C.A., Cannon, R.C., Lattanzio, J.C., et al. 1998, A $\& A$ 332, L17

Gregorio-Monsalvo, I., Gómez, Y., Anglada, G., et al. 2004, ApJ 601, 921

Molster, F.J., Yamamura, I., Waters, L.B.F.M., et al. 1999, Nature 401, 563

Waters, L.B.F.M., Beintema, D.A., Zijlstra, A.A., et al. 1998a, AESA 331, L61

Zijlstra, A.A., Te Lintel Hekkert, P., Pottasch, S.R., et al. 1989, A\&SA 217, 157 